\title{
La présidente rend compte
}

Gabriela Rüttimann

\section{La procédure de consultation \\ devient le modèle de la prescription}

Le vernaculaire dit : « Tout vient à point à qui sait attendre ». Il n’a pas encore été décidé comment le Conseil fédéral réagira aux commentaires sur sa procédure de consultation sur la nouvelle réglementation de la psychothérapie psychologique et si les décennies de mauvaise administration seront réellement corrigées. Comme point faible dans lélaboration de la procédure de consultation, les associations ont clairement critiqué le fait que les associations en tant que représentantes des psychothérapeutes $(\mathrm{h} / \mathrm{f})$ praticiens n’avaient pas été consultées au cours de lélaboration. Le modèle n’a donc pas été développé sur la base de décennies d'expérience pratique et de chiffres clés, mais plutôt sur la base d'un cadre purement quantitatif conçu « autour d'une table des négociations », qui sécarte à bien des égards de la pratique quotidienne. Il y avait aussi une méfiance subliminale à légard des psychothérapeutes $(\mathrm{h} / \mathrm{f})$ en suggérant des activités contraires à la logique, comme, par exemple, l'enregistrement de rapports historiques (de déroulement) qui ne devraient pas être délaissés aux thérapeutes, mais à un médecin qui ne connaît pas du tout le processus thérapeutique.

Il sest avéré très tôt que la procédure de consultation soulèverait un peu de poussière. Malheureusement, certaines des contributions des opposants (h/f) au modèle de la prescription étaient clairement déplacées. Lévénement a ensuite été repris par les médias et les avantages et les inconvénients ont été commentés avec une plume critique. Sur notre site Web www.psychotherapie.ch/Aktuell/ Medien, vous trouverez une chronique de la couverture médiatique, ce qui va de soi.

\section{Sondage auprès des membres}

Nous avons mené un sondage auprès des membres afin de préparer la déclaration en réponse à la procédure de consultation du Conseil fédéral. Il était important pour nous de ne pas rédiger notre opinion au-delà de la base, mais de découvrir le plus précisément possible ce qui préoccupait nos membres. De cette façon, nous avons pu intégrer leurs préoccupations dans la déclaration. Il est impressionnant de constater que plus de $30 \%$ des membres ont participé au sondage et que plus de 1000 commentaires ont été reçus au sujet des onze questions posées. Celles-ci pourraient être regroupées de telle sorte qu’on puisse en tirer des énoncés très clairs. Il est réjouissant de constater qu'il n'y a guère de différences entre les régions linguistiques.

Il était clair pour nous dès le début que nous ne serions jamais en mesure de soulever toutes les questions, les préoccupations et les besoins qui avaient été soulevés. Il est toutefois apparu clairement que la plupart des points de discorde et des demandes correspondaient à nos propres points de vue. Vous trouverez également les résultats du sondage auprès des membres dans ce numéro.

Avec notre avis sur le processus de consultation, nous voulions envoyer un signal aussi fort que possible. Les trois associations ASP, FSP et SBAP ont donc uni leurs forces et se sont mises d'accord. Il était important pour nous de nous présenter avec une position commune et de ne pas nous laisser diviser.

\section{Retraite du comité}

La retraite du comité a également été dominée par la procédure de consultation. Il s'agissait d'analyser l'enquête auprès des membres et de prendre position. Il sest avéré que cette enquête a été très utile et nous a apporté le soutien nécessaire en tant quassociation et en tant que représentants des intérêts des psychothérapeutes $(\mathrm{h} / \mathrm{f})$. Nous avons également pris conscience que nous nous trouvions à un moment historique important.

\section{Mise à jour du code de déontologie}

Avec l'introduction de la LPsy (Loi sur les professions de la psychologie), notre rôle en tant qu'association professionnelle a changé. Cela signifie que diverses réglementations ont également dû être adaptées à la nouvelle situation. En plus du règlement d'admission des nouveaux membres, les codes de déontologie jouent un rôle très important dans notre association, car elles contiennent tous les droits et devoirs importants concernant l'exercice de la profession de psychothérapeute, qui sont obligatoires pour nos membres. Afin de les ventiler et de nous assurer que le contenu est légal, nous avons demandé un soutien juridique.

La révision du code de déontologie a été approuvée par le comité et sera soumise à l'appro-

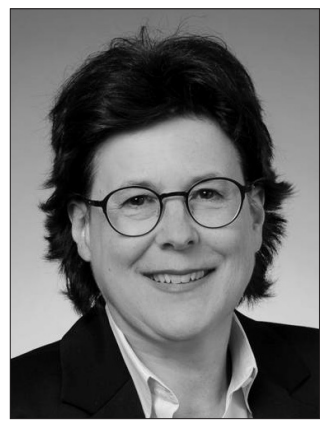


bation de la prochaine assemblée des membres le 17 avril 2020. Jusquà cette date, les codes de déontologie actuels restent en vigueur

\section{Règlement sur la formation continue}

Selon la LPsy et dans le sens de l'assurance qualité, les psychothérapeutes (h/f) sont tenu(e)s de suivre régulièrement une formation continue. Les associations ont décidé conjointement d'harmoniser la formation continue et d'appliquer les mêmes critères. Ce qui est nouveau, c'est que le devoir de formation continue est d'au moins 240 unités de formation continue sur trois ans au lieu de 400 unités sur cinq ans. Comme auparavant, une unité de formation continue dure habituellement 60 minutes, mais au moins 45 minutes. $\mathrm{Au}$ cours de la période de révision de trois ans, au moins deux formes différentes de formation continue doivent être prouvées.

Les membres sont tenus de tenir un dossier de formation continue qui nous sera soumis à la fin de la période d'examen. Sur la base du certificat de formation continue, l'ASP leur délivre un certificat de formation continue.

Le règlement de la formation continue et d'autres documents sont disponibles dans la section réservée aux membres sur notre site Web.

\section{Gabriela Rüttimann est présidente de l'ASP.}

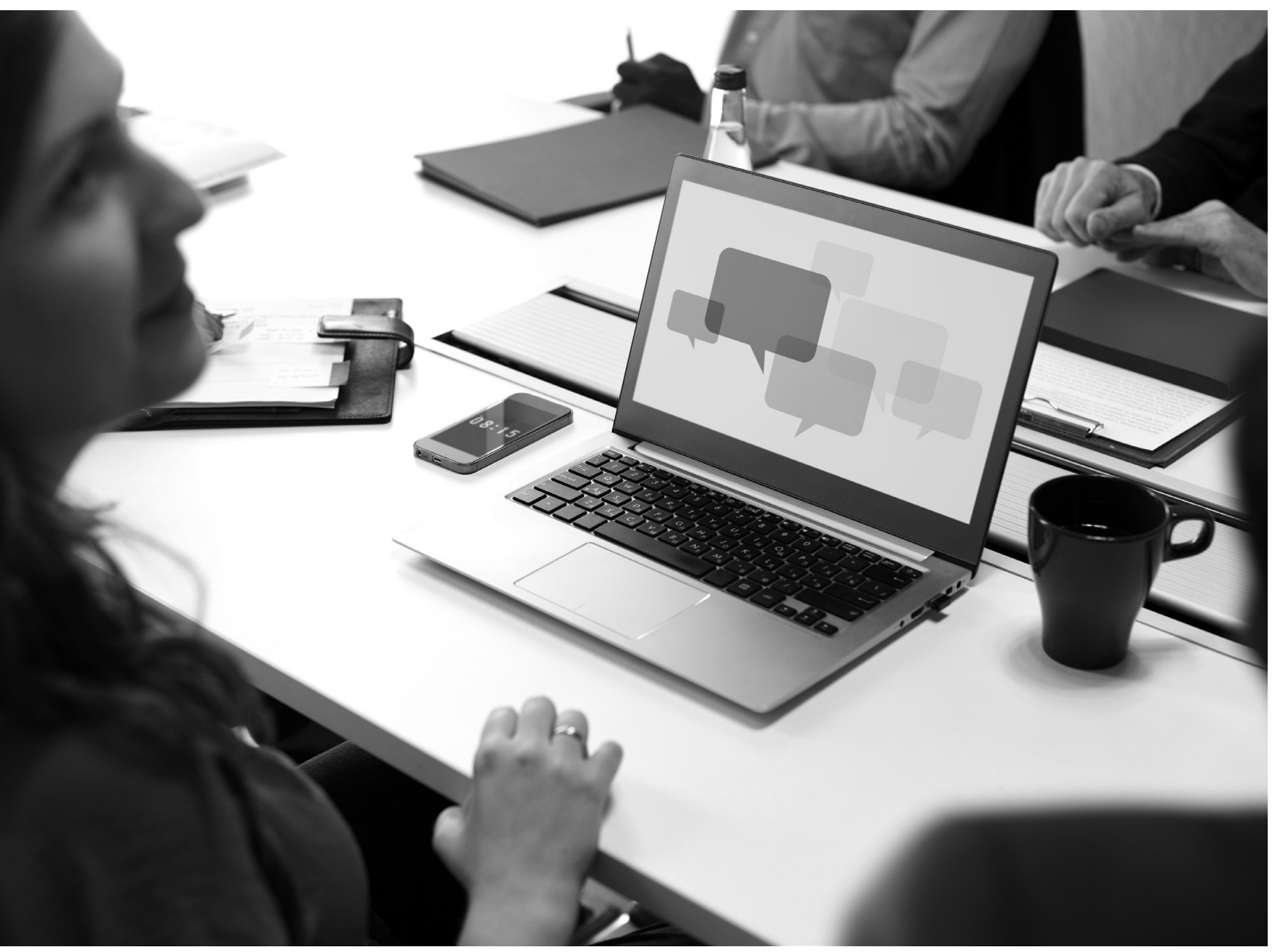

